Bull. Austral. Math. Soc.

28D05, 47A35

VOL. 67 (2003) [39-50]

\title{
COBOUNDARY EQUATIONS OF EVENTUALLY EXPANDING TRANSFORMATIONS
}

\author{
YOUNG-HO AHN
}

Let $T$ be an eventually expansive transformation on the unit interval satisfying the Markov condition. Then $T$ is an ergodic transformation on $(X, \mathcal{B}, \mu)$ where $X=[0,1)$, $\mathcal{B}$ is the Borel $\sigma$-algebra on the unit interval and $\mu$ is the $T$ invariant absolutely continuous measure. Let $G$ be a finite subgroup of the circle group or the whole circle group and $\phi: X \rightarrow G$ be a measurable function with finite discontinuity points. We investigate ergodicity of skew product transformations $T_{\phi}$ on $X \times G$ by showing the solvability of the coboundary equation $\phi(x) g(T x)=\lambda g(x),|\lambda|=1$. Its relation with the uniform distribution $\bmod M$ is also shown.

\section{INTRODUCTION}

Let $(X, \mathcal{B}, \mu)$ be a probability space and $T$ be a measure preserving transformation on $X$. A transformation $T$ on $X$ is called ergodic if the constant function is the only $T$-invariant function and it is called weakly mixing if the constant function is the only eigenfunction with respect to $T$. A measure preserving transformation $T$ is called exact if $\bigcap_{n=0}^{\infty} T^{-n} \mathcal{B}$ is the trivial $\sigma$-algebra consisting of empty set and whole set modulo measure zero sets. So exact transformation are as far from being invertible as possible. Recall that if a transformation is exact then that transformation is weakly mixing ([11]).

A piecewise differentiable transformation $T:[0,1) \rightarrow[0,1)$ is said to be eventually expansive if some iterate of $T$ has its derivative bounded away from 1 in modulus, that is, $\left|\left(T^{n}\right)^{\prime}\right|>1$ everywhere for some $n$. Let $\left\{\Delta_{i}\right\}$ be a countable (or finite) partition of the unit interval $[0,1)$ by subintervals. Suppose that an eventually expansive map $T$ on the interval $[0,1)$ satisfies

(i) $\left.T\right|_{\text {Int } \Delta_{i}}$ has a $C^{2}$-extension to the closure of $\Delta_{i}$,

(ii) $\left.T\right|_{\text {Int } \Delta_{i}}$ is strictly monotone,

(iii) $\overline{T\left(\Delta_{i}\right)}=[0,1]$, and in the case that the number of subintervals in the partition is infinite

(iv) $\sup _{i}\left\{\sup _{x_{1} \in \operatorname{Int} \Delta_{i}}\left|T^{\prime \prime}\left(x_{1}\right)\right| / \inf _{x_{2} \in \operatorname{Int} \Delta_{i}}\left|T^{\prime}\left(x_{2}\right)\right|^{2}\right\}<\infty$.

Received 20th March, 2002

This work was supported by the Brain Korea 21 Project in 2001.

Copyright Clearance Centre, Inc. Serial-fee code: 0004-9727/03 \$A2.00+0.00. 
Then it is well known that there exists a measure $\mu$ which is (a) $T$-invariant, (b) exact, and (c) finite and of the form $d \mu=\rho(x) d x$ where $\rho$ is continuous and $1 / C<\rho<C$ for some $C>0([1,3,4])$.

The conditions of the above fact can be modified in several ways. One modification is called the Markov condition, when the number of sets in the partition is finite and $\overline{T\left(\Delta_{i}\right)}$ is a union of $\overline{\Delta_{j}}$. In this paper, let $\mathbb{T}$ be the unit circle in the complex plane, $\mathbb{T}=\{z \in \mathbb{C}:|z|=1\}$.

In [9], Siboni consider the skew product transformation $T_{2, a, \omega}$ on the torus, $[0,1)$ $\times[0,1)$ defined by

$$
(x, y) \mapsto(2 x, y+a x+\omega) \quad(\bmod 1) .
$$

He proved a criterion for the ergodicity of the transformation by accurate estimation of correlations of characteristic functions.

Let $T$ be an eventually expansive transformation on the unit interval satisfying the Markov conditions. Then $T$ is an exact transformation on $(X, \mathcal{B}, \mu)$ where $X=[0,1)$, $\mathcal{B}$ is Borel $\sigma$-algebra on the unit interval and $\mu$ is the $T$ invariant absolutely continuous measure. Let $G$ be a finite subgroup of the circle group or the whole circle group $\mathbb{T}$ and $\phi: X \rightarrow G$ a measurable function with finite discontinuity points. In this paper we investigate ergodicity of skew product transformations $T_{\phi}$ on $X \times G$ by showing the solvability of the coboundary equation $\phi(x) g(T x)=\lambda g(x),|\lambda|=1$ and we give a simple proof and generalisations of Siboni's results, see Proposition 3.

Let $X=\{x: 0 \leqslant x<1\}$ be the compact group of real numbers modulo 1 , and let $\theta \in X$ be irrational. The numbers $j \theta, j=0, \pm 1, \ldots$, comprise a dense subgroup of $X$. For each interval $I \subset X$ and $n>0$ define $S_{n}=S_{n}(\theta, I)$ to be the number of integers $j, 0 \leqslant j \leqslant n-1$, such that $j \theta \in I$. By the Kronecker-Weyl theorem $\lim _{n \rightarrow \infty} S_{n} / n=\mu(I)$, where $\mu$ is Lebesgue measure on $X([6])$. Veech [10] was interested in the behaviour of the sequence $\left\{d_{n}\right\}$ of parities of $\left\{S_{n}\right\}$, that is, $d_{n}$ is 0 or 1 as $S_{n}$ is even or odd. He investigated the existence of the limit

$$
\mu_{\theta}(I)=\lim _{N \rightarrow \infty} \frac{1}{N} \sum_{n=1}^{N} d_{n},
$$

and he showed that a necessary and sufficient condition for $\mu_{\theta}(I)$ to exist for every interval $I \subset X$ is that $\theta$ has bounded partial quotients. He also showed that $d_{n}$ is evenly distributed if the length of the interval is not an integral multiple of $\theta$ modulo 1 .

In this paper, we are interested in the uniform distribution of the sequence $d_{n}$ $\in\{0, \ldots, M-1\}$ defined by

$$
d_{n}(x) \equiv \sum_{k=0}^{n-1} 1_{E}\left(T^{k} x\right) \quad(\bmod M)
$$


for eventually expanding transformations, particularly for generalised $L$-covering maps (which will be defined in section 3 ) and for Gauss transformation on the interval. Here $1_{E}(x)$ denotes the indicator function of $E \subset X$.

To investigate the sequence $\left\{d_{n}(x)\right\}$, we consider the behaviour of the sequence $\exp \left((2 \pi i / M) d_{n}(x)\right)$ and check whether this sequence is uniformly distributed on the finite group $G$ generated by $\exp ((2 \pi i) / M)$. Weyl's criterion for uniform distribution says that the sequence $\exp \left((2 \pi i / M) d_{n}(x)\right)$ is uniformly distributed if and only if $\lim _{N \rightarrow \infty} \sum_{n=1}^{N} / N \exp ^{k}\left((2 \pi i / M) d_{n}(x)\right)=0$ for all $1 \leqslant k \leqslant M-1([6])$. We investigate this problem from the viewpoint of spectral theory. Let $(X, \mu)$ be a probability space and $T$ be an ergodic measure preserving transformation on $X$, which is not necessarily invertible. Let $\phi(x)$ be a $G$-valued function defined by $\phi(x)=\exp \left((2 \pi i / M) \mathbf{1}_{E}(x)\right)$. Consider the skew product transformation $T_{\phi}$ on $X \times G$ defined by $T_{\phi}(x, g)=(T x, \phi(x) g)$. Then

$$
\lim _{N \rightarrow \infty} \frac{1}{N} \sum_{n=1}^{N} \exp ^{k}\left(\frac{2 \pi i}{M} d_{n}(x)\right) \cdot z^{k}=\lim _{N \rightarrow \infty} \frac{1}{N} \sum_{n=1}^{N} U_{T_{\phi}} f(x, z)
$$

where $U_{T_{\phi}}$ is an isometry on $L^{2}(X \times G)$ induced by $T_{\phi}$ and $f(x, z)=z^{k}$. If $T_{\phi}$ is ergodic, we may apply Birkhoff's Ergodic Theorem to $f$ to deduce that $\lim _{N \rightarrow \infty} 1 / N \sum_{n=1}^{N} \exp ^{k}\left((2 \pi i / M) d_{n}\right.$ $(x))=0$, Recall that the dual group of $G$ consists of the $\gamma_{k}(z)=z^{k}$ for $0 \leqslant k \leqslant M-1$. Hence $L^{2}(X \times G)=\bigoplus_{k=0}^{M-1} L^{2}(X) \cdot z^{k}$ and each $L^{2}(X) \cdot z^{k}$ is an invariant subspace of $U_{T_{\phi}}$. If $f(x, z) \in L^{2}(X, G)$ then $f(x, z)=\sum_{k=0}^{M-1} f_{k}(x) z^{k}$, and

$$
U_{T_{\phi}} f(x, z)=\sum_{k=0}^{M-1} \phi^{k}(x) f_{k}(T x) \cdot z^{k}
$$

Hence, if $f$ is an eigenfunction with eigenvalue $\lambda$ we have $\phi^{k}(x) f_{k}(T x)=\lambda f_{k}(x)$ for all $k$. Recall that a nonconstant function $h(x)$ is called a coboundary if $h(x)=\overline{q(T x)} q(x)$, $|q(x)|=1$ almost everhwyere on $X$.

In [2], Ahn and Choe considered the case when $T$ is an $(1 / L, \ldots, 1 / L)$-Bernoulli transformation and show and that if $E$ is a cylinder set with the same missing initial digit and $M=2$, then the sequence $\left\{d_{n}\right\}$ is evenly distributed. In this paper, we show that for all generalised $L$-covering maps and Gauss transformation on the unit interval, the sequence $\left\{d_{n}\right\}$ is uniformly distributed and that compact group extension by $\phi(x)$ is exact.

\section{COBOUNDARY EQUATIONS}

Let $(Y, \mathcal{C}, \mu)$ be a probability space, $f \in L^{1}(Y, \mathcal{C}, \mu)$ and $\mathcal{B} \subset \mathcal{C}$ a sub $\sigma$-algebra. We denote by $E(f \mid \mathcal{B})$ the conditional expectation of $f$ with respect to $\mathcal{B}$. Recall that this 
is a $\mathcal{B}$-measurable function $g$ with the property that $\int_{B} f d \mu=\int_{B} g d \mu$ for all $B \in \mathcal{B}$. Let $S$ be a transformation defined on $Y$ and $\mathcal{B}$ be exhaustive that is, $S^{-1} \mathcal{B} \subset \mathcal{B}$ and $S^{n} \mathcal{B} \uparrow \mathcal{C}$ as $n \rightarrow+\infty$. The Martingale Convergence Theorem says that $E\left(f \mid S^{n} \mathcal{B}\right)$ converges to $f$ almost everywhere and in $L^{1}(Y, \mathcal{C}, \mu)$ as $n \rightarrow+\infty$ for $f \in L^{1}(Y, \mathcal{C}, \mu)$

LEMMA 1. Let $S$ be a measure preserving transformation on $(Y, \mathcal{C}, \mu)$, and $\mathcal{B}$ be an exhaustive $\sigma$-algebra $\mathcal{B} \subset \mathcal{C}$, and let $\phi: Y \rightarrow \mathbb{T}$ be a $\mathcal{B}$-measurable map to the circle group $\mathbb{T}$. If $q: Y \rightarrow \mathbb{T}$ is a $\mathcal{C}$-measurable solution to the equation $\phi \cdot q \circ S=q$, then $q$ is $\mathcal{B}$-measurable.

Proof: We follow an idea of Parry in [8]. Applying the conditional expectation operator $E(\cdot \mid \mathcal{B})$ to the equation

$$
\phi \cdot q \circ S=q
$$

we obtain $\phi \cdot E(q \circ S \mid \mathcal{B})=E(q \mid \mathcal{B})$ or $\phi \cdot E(q \mid S \mathcal{B}) \circ S=E(q \mid \mathcal{B})$. Multiplying this with the inverse of $(*)$ we have $\overline{q(y)} \cdot E(q \mid \mathcal{B})(y)=\overline{q(S y)} \cdot E(q \mid S \mathcal{B}) \circ S(y)$ almost everywhere so that $\int_{Y} \bar{q} \cdot E(q \mid \mathcal{B}) d \mu=\int_{Y} \bar{q} \cdot E(q \mid S \mathcal{B}) d \mu$. By exactly the same argument, using $S^{n} \mathcal{B}$ in place of $\mathcal{B}$, we have $\int_{Y} \bar{q} \cdot E\left(q \mid S^{n} \mathcal{B}\right) d \mu=\int_{Y} \bar{q} \cdot E\left(q \mid S^{n+1} \mathcal{B}\right) d \mu$ so that $\int_{Y} \bar{q} \cdot E(q \mid$ $\mathcal{B}) d \mu=\int_{Y} \bar{q} \cdot E\left(q \mid S^{n} \mathcal{B}\right) d \mu$. Taking limits, we get $\int_{Y} \bar{q} \cdot E(q \mid \mathcal{B}) d \mu=\int_{Y}|q|^{2} d \mu$. Thus $E(q \mid \mathcal{B})=q$ almost everywhere, and $q$ is $\mathcal{B}$-measurable.

Proposition 1. Let $Y=\prod_{-\infty}^{\infty}\{0,1, \ldots, L-1\}$ where $L \leqslant \infty$ and let $\sigma$ be the shift map on $Y$ with $\sigma$-invariant measure $\mu$. Let $\mathcal{P}$ denote the state partition $\left\{P_{k}: P_{k}\right.$ $=\left\{x: x_{0}=k\right\}$ for $\left.0 \leqslant k \leqslant L-1\right\}$, and let $\mathcal{B}_{l}^{m}=\bigvee_{i=l}^{m} \sigma^{-i} \mathcal{P}$ for $l \leqslant m$. Assume that $\phi(x)$ is a $\mathbb{T}$-valued $\mathcal{B}_{l}^{m}$ measurable function. If $g(x)$ is a $\mathbb{T}$-valued solution of the equation, $\phi(x) g(\sigma x)=g(x)$ then $g(x)$ is also a $\mathcal{B}_{l}^{m}$ measurable function.

Proof: Let $\mathcal{B}=\bigvee_{i=l}^{\infty} \sigma^{-i} \mathcal{P}$. Then $\phi(x)$ is $\mathcal{B}$ measurable and $\mathcal{B}$ is exhaustive with respect to $\sigma$. Since $\phi(x) g(\sigma x)=g(x), g(x)$ is also $\mathcal{B}$-measurable by the above Lemma. Now let $\mathcal{A}=\bigvee_{i=-m}^{\infty} \sigma^{i} \mathcal{P}$. Then $\phi\left(\sigma^{-1} x\right)$ is $\mathcal{A}$ measurable and $\mathcal{A}$ is exhaustive with respect to $\sigma^{-1}$. Since $\phi(x) g(\sigma x)=g(x)$ can be rewritten as $\phi\left(\sigma^{-1} x\right) g(x)=g\left(\sigma^{-1} x\right)$, that is, $\phi\left(\sigma^{-1} x\right) \overline{g\left(\sigma^{-1} x\right)}=\overline{g(x)}, g(x)$ is also $\mathcal{A}$ measurable by applying the above Lemma to the map $\sigma^{-1}$. Hence the conclusion follows.

\section{THE INTERVAL MAPS AND SYMBOLIC DYNAMICS}

In this section we apply the previous result to Markov maps. Consider the behaviour of the iterates of a map $\tau$ of the unit interval to itself. We also assume that $\tau$ is noninvertible and piecewise continuous. Here are several well-known examples:

(a) $\tau(x)=2 x(\bmod 1)$; 
(b) $\quad \tau(x)=\beta x(\bmod 1), \beta=(1+\sqrt{5}) / 2$;

(c) Gauss transform $\tau(x)=1 / x(\bmod 1)$.

For each of these transformations explicit formulas are known for absolutely continuous invariant measures:

(a) the Lebesgue measure $d x$;

(b) $d \mu=\beta d x, 0 \leqslant x<\beta^{-1}$, and $d \mu=d x, \beta^{-1} \leqslant x<1$;

(c) the Gauss measure $d \mu=(1 / \log 2) d x /(1+x)$.

Definition 1: Let $\tau:[0,1) \rightarrow[0,1) 0=a_{0}<a_{1}<\cdots<a_{L}=1$, and let $\left\{I_{j}\right\}_{j=0}^{L-1}$ be a partition of $[0,1)$ with $I_{j}=\left[a_{j}, a_{j+1}\right), 0 \leqslant j \leqslant L-1 \leqslant \infty$. Assume that $\tau$ satisfies

(1) $\left.\tau\right|_{\text {Int } I_{i}}$, the restriction of $\tau$ to interior points of $I_{i}$, has a $C^{2}$-extension to the closure of $I_{i}$,

(2) $\left.\tau\right|_{\text {Int } I_{i}}$ is strictly monotone,

(3) $\overline{\tau\left(I_{i}\right)}=[0,1]$, and, in the case where $L=\infty$

(4) $\sup _{i}\left\{\sup _{x_{1} \in \operatorname{Int} I_{i}}\left|\tau^{\prime \prime}\left(x_{1}\right)\right| / \inf _{x_{2} \in \operatorname{Int} I_{i}}\left|\tau^{\prime}\left(x_{2}\right)\right|^{2}\right\}<\infty$.

Suppose that for some $n,\left|d \tau^{n} / d t\right| \geqslant \theta>1$ for all $t$. If we regard the above map $\tau$ as being defined on the unit circle, its winding number equals $L$. We call it a generalised $L$-covering map.

It is known that $\tau$ has a finite ergodic measure $\rho(x) d x$ where $\rho(x)$ is piecewise continuous and $1 / D<\rho<D$ for some $D>0$. See $[1,3]$.

Given a generalised $L$-covering map $\tau$, construct an one-sided shift space on $L$ symbols as follows: To each $t \in[0,1)$ there corresponds a one-sided infinite sequence $\left[a_{0}, a_{1}, \ldots, a_{n}, \ldots\right]$ such that $\tau^{n}(t) \in I_{a_{n}}$. For some $t \in[0,1)$, we can find $N$ such that its representation $t=\left[a_{0}, a_{1}, \ldots, a_{n}, \ldots\right]$ satisfies the condition that $a_{n}=0$ for all $n \geqslant N$. We call such a $t$ a generalised $L$-adic point. Let $X$ be the set of all such sequences and $\psi$ be the assignment of a sequence to a point. Since $\tau$ has a finite absolutely continuous ergodic measure $\rho(t) d t$, we can define a shift invariant measure $\nu$ on any cylinder set $C \subset \prod_{0}^{\infty}\{0,1, \ldots, L-1\}$ by $\nu(C)=\int_{\psi^{-1}(C)} \rho(x) d x$. Note that $\psi^{-1}(C)$ is a union of intervals with generalised $L$-adic endpoints. The Kolmogorov Extension Theorem guarantes that $\nu$ may be uniquely extended to the whole $\sigma$-algebra. We call the shift space $X$ the $L$-adic symbolic system obtained from $\tau$. Recall that two measure preserving transformations $T_{1}$ and $T_{2}$ on $X_{1}$ and $X_{2}$ are said to be isomorphic if there exists a measure preserving transformation $\psi: X_{1} \rightarrow X_{2}$ which is one-to-one such that $\psi \circ T_{1}=T_{2} \circ \psi$ on $X_{1}$ modulo sets of measure zero. The mapping $\phi$ introduced above is an isomorphism between $((0,1), \rho d t, \tau)$ and the one-sided shift space $(X, d \nu, \sigma)$.

Our construction also applies even if the condition (3) in Definition 1 does not hold. For example, the interval map $x \mapsto \beta x(\bmod 1), \beta=(1+\sqrt{5}) / 2$ has the following special property: Put $I_{0}=\left[0, \beta^{-1}\right), I_{1}=\left[\beta^{-1}, 1\right)$. If $x \in I_{1}$, then $\tau x \in I_{0}$. In other words, in 
any sequence $\left[x_{0}, x_{1}, \ldots, x_{n}, \ldots\right]$ the symbol 1 does not occur consecutively. Hence in this case $X$ would not be the full shift $\prod_{0}^{\infty}\{0,1\}$. In fact, it is a shift of finite type with a forbidden block 11 . See [1].

Definition 2: An $L$-adic multi-index $\vec{n}$ is a finite sequence of elements of $\{01 \ldots$, $L-1\}$ and will denote by $\vec{n}=\left\langle n_{1}, \ldots, n_{k}\right\rangle$ Its length $k$ is denoted by $|\vec{n}|$. If there is no danger of ambiguity we call it a multi-index. If $|\vec{n}|=1$, then $\vec{n}=\left\langle n_{0}\right\rangle$ for some $n_{0}$, and we write $\vec{n}=n_{0}$.

Let $\tau$ be a map as given in Definition 1. Define $h_{i}:[0,1) \rightarrow[0,1)$ by letting $h_{i}(t)$ be the unique element in the set $\tau^{-1}(\{t\}) \cap I_{i}, i=0,1, \ldots L-1$. Note that $\tau^{-1}(\{t\})$ $=\left\{h_{0}(t), h_{1}(t), \ldots, h_{L-1}(t)\right\}$. For a multi-index $\vec{n}=\left\langle n_{1}, \ldots, n_{k}\right\rangle$, define $h_{\vec{n}}=h_{n_{1}} \circ \ldots \circ$ $h_{n_{k}}$.

For example, consider the transformation $\tau(x)=2 x(\bmod 1)$ defined on the unit interval with the partition $\{[0,(1 / 2)),[(1 / 2), 1)\}$. Since every $t \in[0,1)$ can be represented as a binary expansion, say $t=\left[t_{1}, t_{2}, \ldots\right], h_{0}(t)=(1 / 2) t=\left[0, t_{1}, t_{2}, \ldots\right]$ and $h_{1}(t)$ $=(1 / 2)+(1 / 2) t=\left[1, t_{1}, t_{2}, \ldots\right]$. Hence $h_{i}(t)=\left[i, t_{1}, t_{2}, \ldots\right]$ for $i$ $=0,1$. So $h_{\vec{n}}(t)=\left[n_{1}, \ldots, n_{k}, t_{1}, t_{2}, \ldots\right]$ where $\vec{n}=\left\langle n_{1}, \ldots, n_{k}\right\rangle$. In particular, $h_{\vec{n}}(0)=\left[n_{1}, \ldots, n_{k}\right]=\sum_{j=1}^{k} n_{j} 2^{-j}$.

From Definitions 1 and 2 we easily obtain

LEMma 2. Put $\vec{n}=\left\langle n_{1}, \ldots, n_{k}\right\rangle$. Let $\tau$ and $h_{i}$ be as given in Definitions 1 and 2 . Then

(1) $\tau^{-k}(\{t\})=\left\{h_{\vec{n}}(t):|\vec{n}|=k\right\}$,

(2) $\tau^{k}\left(h_{\vec{n}}(x)\right)=x$ where $k=|\vec{n}|$,

(3) $h_{\vec{n}}\left(\left[a_{1}, a_{2}, \ldots\right]\right)=\left[n_{1}, \ldots, n_{k}, a_{1}, a_{2}, \ldots\right]$, and

(4) $\tau^{-k}(E)=\bigcup_{|\vec{n}|=k} h_{\tilde{n}}(E)$ for any subset $E$.

For any fixed integer $k>0$ let $\mathcal{P}_{0}^{k}$ be the set of numbers of the form $\left[a_{1}, \ldots, a_{k}\right]$, $a_{i}=0,1, \ldots, L-1$ so that the points in $\mathcal{P}_{0}^{k}$ partition the whole interval $[0,1)$ into $L^{k}$ segments. Then (i) $h_{\vec{n}}([0,1))$ is one of the $L^{k}$ intervals obtained by partitioning the unit interval by the points in $\mathcal{P}_{0}^{k}, k=|\vec{n}|$ and (ii) if $x \in h_{\vec{n}}([0,1)), \vec{n}=\left\langle n_{1}, \ldots, n_{k}\right\rangle$, then the coded sequence for $x$ is $\left[n_{1}, \ldots, n_{k}, \ldots\right]$.

Proposition 2. A complex valued step function $\phi(x)$ with finite generalised $L$-adic discontinuity points $a_{1} \leqslant t_{1}<\ldots<t_{n}<1$, is not a coboundary for any generalised $L$-covering map.

Proof: Let $\left(X, \sigma_{X}, \nu\right)$ be the one-sided shift space which is isomorphic to the given $L$-covering map. Let $Y=\prod_{-\infty}^{\infty}\{0,1, \ldots, L-1\}, \sigma_{Y}$ the two-sided shift and $\mu$ the unique measure on $Y$ so that $\left(Y, \sigma_{Y}, \mu\right)$ is the natural extension of $\left(X, \sigma_{Y}, \mu\right)$ 
Assume that $\phi(x) h(\tau x)=h(x)$. Since $\phi(x)$ is step function with finite $L$-adic discontinuity points, we can regard $\phi(x)$ is function on $Y$ which is measurable, with respect to $\mathcal{B}_{0}^{m}=\bigvee_{i=0}^{m} \sigma^{-i} \mathcal{P}$ for some $m<\infty$ (Lemma 2). Hence $h(x)$ is also $\mathcal{B}_{0}^{m}$-measurable by Proposition 1. Thus $h(x)$ is also a step function with finite $L$-adic discontinuity points. Hence there exists $0<r \leqslant a_{1}$ such that $h(x)$ is constant on $[0, r)$. Thus $\phi(x) h(x)=h(x)$ on $[0, r)$, that is, $\phi(x)=1$ on $\left[0, t_{1}\right)$. Since $\phi(x) h(\tau x)=h(x), t_{1} \geqslant a_{1}$ and $\tau\left[0, a_{1}\right)=[0,1)$, $h(x)$ is constant on $[0,1)$. Hence the conclusion follows.

Example 1. For a transformation $T:[0,1) \rightarrow[0,1)$ defined by $x \mapsto 2 x(\bmod 1)$, we consider the following. Let $I=[(3 / 4), 1), F=\bigcup_{k=0}^{\infty}\left(1 / 2^{k}\right) I$ and $E=F \Delta T^{-1} F$. Then $\phi(x)=\exp \left(\pi i 1_{E}(x)\right)$ is a coboundary even if the discontinuity points of $\phi(x)$ are contained in $[(1 / 2), 1)$ where the cobounding function $h(x)=\exp \left(\pi i 1_{F}(x)\right)$. Hence the assumption in Proposition 2 of finite discontinuity points cannot be dropped.

\section{A CLASS OF SKEW PRODUCTS OF CIRCLE ENDOMORPHISMS}

In this section, we investigate the dynamical properties of a class of skew products of circle endomorphisms.

Definition 3: For a positive integer $L$, let $T_{L, a, w}$ be the skew-product transformation on the torus, $[0,1) \times[0,1)$ defined by

$$
(x, y) \mapsto(L x, y+a x+\omega) \quad(\bmod 1)
$$

In [9], Siboni considered the skew product transformation $T_{2, a, \omega}$ and proved a criterion of ergodicity of the transformation by the accurate estimation of correlations of characteristic functions. In this section, we shall give a simple proof and generalisation of his results.

For a fixed natural number $L$, let $T$ be the transformation on $X=[0,1)$ defined by $T x=L x(\bmod 1)$.

LEMMA 3. Let $S$ and $S^{\prime}$ be transformations of the torus defined by

$$
\begin{aligned}
& S(x, y)=(L x, y+a x) \quad(\bmod 1) \quad \text { and } \\
& S^{\prime}(x, y)=\left(L x, y+\sum_{k=0}^{L-1} \frac{k a}{L-1} 1_{[(k / L),(k+1 / L))}(x)\right) \quad(\bmod 1) .
\end{aligned}
$$

Then $S$ and $S^{\prime}$ are isomorphic.

Proof: We will use the $L$-adic expansion of $x ; x=\left(x_{0} / L\right)+\left(x_{1} / L^{2}\right)+\cdots$. Let $\phi(x, y)=(x, y+(a / L-1) x)(\bmod 1)$. Then $\phi^{-1}(x, y)=(x, y-(a / L-1) x)(\bmod 1)$ 
and

$$
\begin{aligned}
\phi^{-1} S \phi(x, y) & =\phi^{-1}\left(L x, y+a x+\frac{a}{L-1} x\right) \\
& =\left(L x, y+a x+\frac{a}{L-1} x-\frac{a}{L-1}(L x-k)\right) \quad \text { if } x_{0}=k \\
& =\left(L x, y+\frac{k a}{L-1}\right) \quad \text { if } x_{0}=k \\
& =S^{\prime}(x, y) .
\end{aligned}
$$

Hence to investigate the spectral type of $S$, we only need to study the spectral type of $S^{\prime}$.

PROPOSITION 3. $T_{L, a, \omega}$ is weakly mixing if and only if $a$ is irrational. Further if $a$ is rational then $T_{L, a, \omega}$ is ergodic if and only if $\omega$ is irrational.

Proof: For convenience, let us denote the 2-torus by $X \times \mathbb{T}$. As before, we use the $L$-adic expansion of $x$. Recall that $L^{2}(X \times \mathbb{T})=\bigoplus_{n=-\infty}^{\infty} f(x) \cdot z^{n}$.

Let $\eta(x)=\exp (2 \pi i(a x+\omega)), \psi(x)=\exp (2 \pi i a x)$ and $\phi(x)=\exp \left(2 \pi i a\left(x_{0} / L-1\right)\right)$. Then

$$
U_{T_{n}}\left(f(x) \cdot z^{n}\right)=\exp (2 \pi i n a x) \cdot \exp (2 \pi i n \omega) \cdot f(T x) \cdot z^{n} .
$$

We consider the operator

$$
U(f(x))=\exp (2 \pi i n a x) \cdot \exp (2 \pi i n \omega) \cdot f(T x) .
$$

If $n=0$, then $U(f(x))=f(T x)$. Thus if $f(T x)=\lambda f(x)$ then $f(x)$ is constant and $\lambda=1$ by the mixing property of $T$. Hence it remains to consider the case $n \neq 0$. Assume that $U(f(x))=\lambda f(x)$. Then

$$
\exp (2 \pi i n a x) \exp (2 \pi i n \omega) f(T x)=\lambda f(x)
$$

and $|\lambda|=1$. Without loss of generality we may assume that $|f(x)|=1$ almost everywere. So $\exp (2 \pi i n a x)=\lambda^{\prime} \overline{f(T x)} f(x)$, where $\lambda^{\prime}=\lambda \exp (-2 \pi i n \omega)$. By Lemma $3, U_{T_{\psi}}$ is spectrally equivalent to $U_{T_{\phi}}$ and $U_{T_{\phi}}$ also has an eigenfunction $g(x)$ with eigenvalue $\lambda^{\prime}$, that is, $\overline{\lambda^{\prime}} \exp \left(2 \pi i n \sum_{k=0}^{L-1}(k a / L-1) \mathbf{1}_{[(k / L),(k+1 / L))}(x)\right)=\overline{g(T x)} g(x)$. If $a$ is an irrational number, then there exists the only integer $n$ for mention.

$$
\overline{\lambda^{\prime}} \exp \left(2 \pi i n \sum_{k=0}^{L-1} \frac{k a}{L-1} \mathbf{1}_{[(k / L),(k+1 / L))}(x)\right)
$$

is constant is $n=0$. Hence by Proposition 2, there exists no eigenfunction for $U_{T_{\phi}}$. If $a$ is rational then there exists $n$ such that $\exp \left(2 \pi i n \sum_{k=0}^{L-1}(k a / L-1) 1_{((k / L),(k+1 / L))}(x)\right)=1$. 
So the problem is reduced to finding $g(x)$ such that $g(T x)=\lambda^{\prime} g(x)$. By the mixing property of $T$ we know that $g(x)$ is constant and $\lambda^{\prime}=\lambda \exp (-2 \pi i n \omega)=1$ for each $n \neq 0$. So $U_{T_{\phi}}$ has eigenfunction $g(x, z)=z^{n}$ with eigenvalue $\lambda=\exp (2 \pi i n \omega)$. Hence $T_{L, a, \omega}$ is not weakly mixing. For the ergodicity, we need only consider the case $\lambda=1$, that is, $\exp (2 \pi i n \omega)=1$. Hence if $a$ is rational, then $T_{L, a, \omega}$ is ergodic if and only if $\omega$ is irrational.

REMARK 1. Indeed we have shown that if $a$ is irrational then $T_{L, a, \omega}$ is exact on the torus. In fact, $T_{L, a, \omega}$ is strong mixing. To see this, let $\mathcal{B}_{1}$ be the Borel $\sigma$-algebra on $[0,1)$, $\mathcal{B}_{2}$ be the Borel $\sigma$-algebra on $\mathbb{T}$ and $\mathcal{B}_{\infty}=\bigcap_{n=0}^{\infty} T_{L, a, \omega}^{-n}\left(\mathcal{B}_{1} \times \mathcal{B}_{2}\right)$. We only need to show that $L^{2}\left(\mathcal{B}_{\infty}\right)$ is the set of constant functions. We use the fact that $T_{L, a, \omega}$ commutes with the circle action $(x, z) \mapsto(x, z \cdot g)$ so that for every $n$ the $\sigma$-algebra $T_{L, a, \omega}^{-n}\left(\mathcal{B}_{1} \times \mathcal{B}_{2}\right)$ is preserved by the circle action. Hence $\mathcal{B}_{\infty}$ is invariant with respect to the circle action. If $f \in L^{2}\left(\mathcal{B}_{\infty}\right)$ then it has a representation

$$
f(x, z)=\sum_{k} f_{k}(x) \cdot z^{k}
$$

and $f(x, z \cdot g)=\sum_{k} f_{k}(x) \cdot z^{k} \cdot g^{k}$ for all $g \in \mathbb{T}$. Hence $f_{k}(x) \cdot z^{k} \in L^{2}\left(\mathcal{B}_{\infty}\right)$. So $\left|f_{k}(x) \cdot z^{k}\right|=\left|f_{k}(x)\right|$ is also $\mathcal{B}_{\infty}$ measurable. But $\left|f_{k}(x)\right|$ depends only on $x$. Hence $\left|f_{k}(x)\right|$ is constant by the exactness of $T$. By a similar argument $\overline{f_{k}} \cdot f_{k} \circ T_{L, a, w}$ is also constant, that is,

$$
\exp (2 \pi i k a x) \cdot \exp (2 \pi i k \omega) \cdot f_{k}(T x) \cdot z^{k}=\lambda f_{k}(x) \cdot z^{k}
$$

where $\lambda \in \mathbb{C}$. Thus if $T_{L, a, \omega}$ is weakly mixing then $T_{L, a, \omega}$ is exact. For more information on this subject, see [5].

\section{MOD $M$ NORMALITY OF $L$-COVERING MAPS}

In this section, let $G$ be the finite subgroup of $\mathbb{T}$ generated by $\exp (2 \pi i / M)$.

PROPOSITION 4. Let $T$ be an ergodic transformation on $X$ and $\phi(x)$ be a $G$-valued function. Let $T_{\phi}$ be the skew product transformation defined by $T_{\phi}(x, g)$ $=(T x, \phi(x) \cdot g)$ on $X \times G$. If $\phi(x) h(T x)=h(x)$, then there exists a $G$-valued function $q(x)$ such that the following diagram commutes

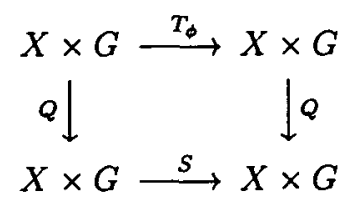

where $Q(x, g)=(x, q(x) \cdot g)$ and $S(x, g)=(T x, g)$. Hence $T_{\phi}$ has $M$ ergodic components. 
Proof: Since $(\phi(x))^{M}=1,(\phi(x))^{M}(h(T x))^{M}=(h(x))^{M}$ is equivalent to $(h(T x))^{M}$ $=(h(x))^{M}$. So we may assume that $(h(x))^{M}=1$ by the ergodicity of $T$. Hence there exist a $G$-valued function $q(x)$ such that $\phi(x) q(T x)=q(x)$. For $q(x)$, of this for it is easy to see that the diagram commutes.

LEMMA 4. Let $\tau$ be piecewise, twice continuously differentiable and such that $\inf _{x \in J_{1}}\left|\tau^{\prime}(x)\right|>1$ where $J_{1}=\left\{x \in X: \tau^{\prime}(x)\right.$ exists $\}$. If the number of discontinuity points of $\tau$ of $\tau^{\prime}$ is finite, then there is a finite collection of sets $L_{1}, \ldots, L_{n}$ and a set of invariant functions $\left\{f_{1}, \ldots, f_{n}\right\}$ such that

(1) each $L_{i}(1 \leqslant i \leqslant n)$ is a finite union of closed intervals;

(2) $L_{i} \cap L_{j}$ contains at most a finite number of points when $i \neq j$;

(3) $f_{i}(x)=0$ for $x \notin L_{i}, 1 \leqslant i \leqslant n$, and $f_{i}(x)>0$ for almost everywhere $x$ in $L_{i}$

(4) $\int_{L_{\imath}} f_{i}(x) d x=1$ for $1 \leqslant i \leqslant n$;

(5) every $\tau$ invariant function can be written as $f=\sum_{i=1}^{n} a_{i} f_{i}$ with suitable chosen $\left\{a_{i}\right\}$.

Proof: For the proof, See [7].

PRoposition 5. If a $G$-valued function $\phi(x)$ is a step function with finite discontinuity points $a_{1} \leqslant t_{1}<\cdots<t_{n}<1$, then $\phi(x)$ is not coboundary for any generalised L-covering map.

PROOF: Assume that $\phi(x) h(T x)=h(x)$. Without loss of generality assume that $X=[0,1)$. Since $X \times G=\bigcup_{k=0}^{M-1}\{X \times \exp ((2 k \pi i) / M)\}$, we may identify $\{X \times \exp ((2 k \pi i) / M)\}$ with the unit interval $[k, k+1), 0 \leqslant k<M$. Since $\phi(x)$ is a $G$-valued step function with finite discontinuity points, we can regard $T_{\phi}$ as a piecewise continuous map on $[0, M)$ satisfying the condition of Lemma 4 . So we can say that $h(x)$ is also a $G$-valued step function with finite discontinuity points by Lemma 4 and Proposition 4. Hence there exists $0<r \leqslant a_{1}$ such that $h(x)$ is constant on $[0, r)$. Thus $\phi(x) h(x)=h(x)$ on $[0, r)$. So $h(x)$ is constant on $[0,1)$ by the argument of the proof of Proposition 2. The conclusion follows.

REMARK 2. In Proposition 5, we have not assumed that the discontinuity points are generalised $L$-adic points, but rather that the range of $\phi(x)$ is contained in a finite subgroup of $\mathbb{T}$. For generalised $L$-covering maps, mod $M$ normality holds for finite unions of intervals, when the associated step function $\phi(x)$ step satisfies the condition of Proposition 5. Indeed we may also show that the skew product transformation induced by $\phi(x)$ is also exact on $X \times G$, as in fact strong mixing, using the similar arguments, to those in Remark 1. 


\section{MOD $M$ NORMALITY OF GaUSS TRANSFORMATION}

Recall that the Gauss transformation $T$ on $[0,1)$ is defined by

$$
T(x)=\left\{\begin{array}{lll}
0 & \text { if } \quad x=0 \\
\frac{1}{x} & \text { if } \quad x \neq 0 \quad(\bmod 1) .
\end{array}\right.
$$

It is well known that $T$ preserves the Gauss measure on $[0,1)$ given by

$$
\mu(A)=\frac{1}{\log 2} \int_{A} \frac{1}{1+x} d x .
$$

Let $\mathcal{P}=\left\{P_{j}\right\}$ be a partition on $[0,1)$ defined by $P_{j}=[(1 / j+1),(1 / j))$ for $j \in \mathbb{N}$.

Proposition 6 . Let $\left\{B_{i}\right\}$ be a sequence of intervals of $[0,1)$ with rational endpoints and $\left\{b_{i}\right\}$ be a sequence of real numbers. Then a nonconstant function $\phi(x)$ $=\exp \left(2 \pi i \sum_{i=1}^{n} b_{i} \mathbf{1}_{B_{i}}(x)\right)$ is not a coboundary for the Gauss transformation.

Proof: Let $Y=\prod_{-\infty}^{\infty}\{1,2, \ldots\}$ and $Y^{+}=\prod_{0}^{\infty}\{1,2, \ldots\}$. Consider the following commutative diagram

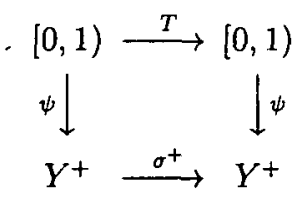

where $(\psi(x))_{i}=j$ if $T^{i} x \in P_{j}$ for $i \in \mathbb{N}$. Then $\psi$ is a an isomorphism between $([0,1), T, \mu)$ and $\left(Y^{+}, \sigma^{+}, \nu^{+}\right)$where $\nu^{+}$is the induced measure by $\psi$ and $\sigma^{+}$is the one-sided shift map on $Y^{+}$. Let $(Y, \sigma, \nu)$ be the natural extension of $\left(Y^{+}, \sigma^{+}, \nu^{+}\right)$where $\sigma$ is the twosided shift map on $Y$. If $\phi(x) g(T x)=g(x)$ then $g(x)$ is also step function with rational discontinuity points and there exist an interval $I$ with rational end points such that $g(x)$ is constant on $I$ by the arguments of Proposition 1 . Since $T^{n} I=[0,1)$ for some $n$, $\phi(x)$ is a function with finite discontinuity points, and $\phi(x) g(T x)=g(x), g(x)$ is also a function with finite discontinuity points. Since $\phi(x) g(T x)=g(x)$ can be rewritten as $\phi(x)=g(x) \overline{g(T x)}, \phi(x)$ must be a function with infinite discontinuity points. this is a contradiction.

Remark 3. By Proposition $6, \bmod M$ normality holds for finite union of intervals with rational end points on Gauss transformation. By the similar arguments as in Remark 1, the induced skew product transformation on $X \times G$ is also exact.

\section{REFERENCES}

[1] R. Adler and L. Flatto, 'Geodesic flows, interval maps and symbolic dynamics', Bull. Amer. Math. Soc. 25 (1991), 229-334. 
[2] Y. Ahn and G.H. Choe, 'Spectral types of skewed Bernoulli shift', Proc. Amer. Math. Soc. 128 (2000), 503-510.

[3] T. Bedford, M. Keane and C. Series(eds.), Ergodic theory, symbolic dynamics and hyperbolic space (Oxford Univ. Press, New York, 1991), pp. 107-109.

[4] A. Boyarsky and P. Góra, Laws of chaos (Birkhäuser, Boston MA, 1997).

[5] Z. Coelho and W. Parry, 'Shift endomorphisms and compact Lie extensions', Bol. Soc. Brasil. Mat. (N.S.) 29 (1998), 163-179.

[6] E. Hlawka, The theory of uniform distribution (A B Academic publishers, Berkhamsted, 1984).

[7] T. Li and J.A. Yorke, 'Ergodic transformations from an interval into itself', Trans. Amer. Math. Soc. 235 (1978), 183-192.

[8] W. Parry, 'A Cocycle equation for shift', Contemp. Math. 135 (1992), 327-333.

[9] S. Siboni, 'Ergodic properties of a class of skew-systems obtained by coupling the transformation of the 1-torus with the endomorphism $2 x \bmod [0,1['$, Nonlinearity 7 (1994), 1133-1141.

[10] W.A. Veech, 'Strict ergodicity in zero dimensional dynamical system and the Kronecker-Weyl theorem mod 2', Trans. Amer. Math. Soc. 140 (1969), 1-33.

[11] P. Walters, An introduction to Ergodic theory (Springer-Verlag, New York, 1982).

School of Mathematical Sciences

Seoul National University

Seoul 151-747

Korea

e-mail: ahn@euclid.kaist.ac.kr 